\title{
Direct observation of electronic and nuclear ground state splitting in external magnetic field by inelastic neutron scattering on oxidized ferrocene and ferrocene containing polymers
}

\author{
Markus Appel ${ }^{1,2, a}$, Bernhard Frick ${ }^{1}$, Johannes Elbert $^{3}$, Markus Gallei ${ }^{3}$ and Bernd Stühn² \\ ${ }^{1}$ Institut Laue-Langevin, 71 Avenue des Martyrs, CS 20156, 38042 Grenoble Cedex 9, France \\ 2 Institute of Condensed Matter Physics, TU Darmstadt, Hochschulstraße 8, 64289 Darmstadt, Germany \\ ${ }^{3}$ Ernst-Berl Institute for Chemical Engineering and Macromolecular Science, TU Darmstadt, Alarich-Weiss Straße 4, \\ 64287 Darmstadt, Germany
}

\begin{abstract}
The quantum mechanical splitting of states by interaction of a magnetic moment with an external magnetic field is well known, e.g., as Zeeman effect in optical transitions, and is also often seen in magnetic neutron scattering. We report excitations observed in inelastic neutron spectroscopy on the redox-responsive polymer poly(vinylferrocene). They are interpreted as splitting of the electronic ground state in the organometallic ferrocene units attached to the polymer chain where a magnetic moment is created by oxidation. In a second experiment using high resolution neutron backscattering spectroscopy we observe the hyperfine splitting, i.e., interaction of nuclear magnetic moments with external magnetic fields leading to sub- $\mu \mathrm{eV}$ excitations observable in incoherent neutron spin-flip scattering on hydrogen and vanadium nuclei.
\end{abstract}

\section{Introduction}

The experiments presented in this paper were performed in the framework of our project to investigate the ring rotation dynamics of ferrocene containing polymers. These materials belong to the class of stimuli-responsive polymers, which, under the influence of external triggers, are in general able to change their solubility or conformation, or can form and break covalent bonds [1]. In contrast to light, temperature or $\mathrm{pH}$, the redox-stimulus is one of the less studied triggers [2,3]. Redox-responsive polymers can be attained, e.g., by incorporating the redoxactive metal-organic ferrocene sandwich complex into a polymer structure [4]. These polymers have been used, amongst other examples, in self-healing materials [5], for switching surface wettability [6], or ion-selective membrane gating [7].

Incoherent quasielastic neutron scattering is the ideal experimental technique to study the reorientational ring rotation dynamics of the cyclopentadienyl rings of the ferrocene molecule $[8,9]$. We have performed such experiments to compare the ring rotation dynamics in ferrocene and ferrocenium triiodide with oxidized ferrocene complexes, as well as to redox-active poly(vinylferrocene) in oxidized and non-oxidized form. The publication of these experiments is currently in progress.

In this paper we present the results of inelastic neutron scattering experiments on oxidized poly(vinylferrocene) under external magnetic fields. While ferrocene itself is diamagnetic, paramagnetic and even ferromagnetic susceptibilities have been reported for ferrocene containing polymers $[10,11]$. In the case presented here, the oxidation

a e-mail: appel@ill.eu with iodine causes one unpaired electron leading to a magnetic moment on the ferrocene complex. A description of the electronic ground state of ferrocenium cations can be obtained from molecular orbital theory [12]. In our experiments, the quasielastic scattering originating from the ring rotation dynamics proved to be insensitive to the external magnetic field up to $2.5 \mathrm{~T}$, but an inelastic excitation was observed in oxidized poly(vinylferrocene) which we interpret as Zeeman splitting of the electronic magnetic moment.

Likewise, high resolution neutron backscattering experiments under external magnetic fields did not reveal a magnetic field dependence of the quasielastic scattering. However, with the high energy resolution the hyperfine splitting of hydrogen is resolvable and leads to significant broadening of the effective instrumental resolution function. While we are not aware of comparable measurements in external magnetic fields, hyperfine splitting is commonly used to probe the strong internal field in ferromagnetic materials [13,14].

\section{Experimental}

$\mathrm{FcI}_{3}$ was obtained by stoichiometric mixing of ferrocene (Alfa Aesar, purity 99\%) and iodine in dichloromethane and subsequent evaporation of the solvent yielding a black powder. PVFc $\left(20 \mathrm{~kg} \mathrm{~mole}^{-1}\right)$ was synthesized by anionic polymerization according to procedures described in literature [15]. Oxidation of PVFc was obtained by adding iodine to a solution of PVFc in THF in a 1:1 molar ratio of $\mathrm{I}_{2}$ to $\mathrm{Fc}$ units, subsequent precipitation in diethyl ether and drying in vacuo. As the distribution and character of iodine counterions (e.g., $\mathrm{I}^{-}$or $\mathrm{I}_{3}^{-}$) was not investigated

This is an Open Access article distributed under the terms of the Creative Commons Attribution License 4.0, which permits unrestricted use, distribution, and reproduction in any medium, provided the original work is properly cited. 


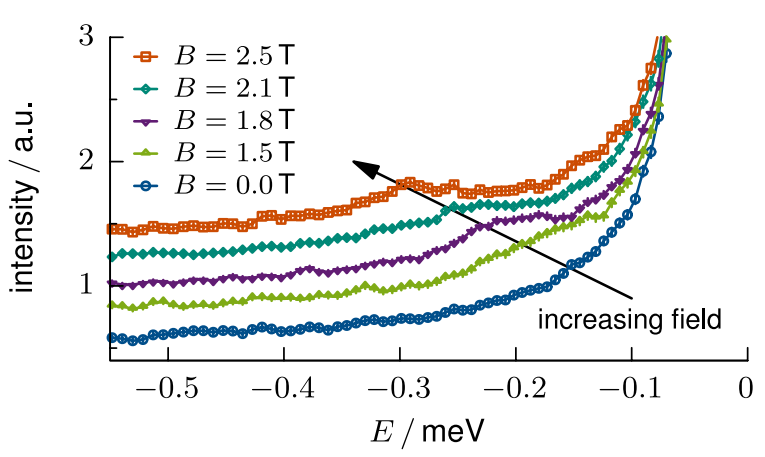

(a)

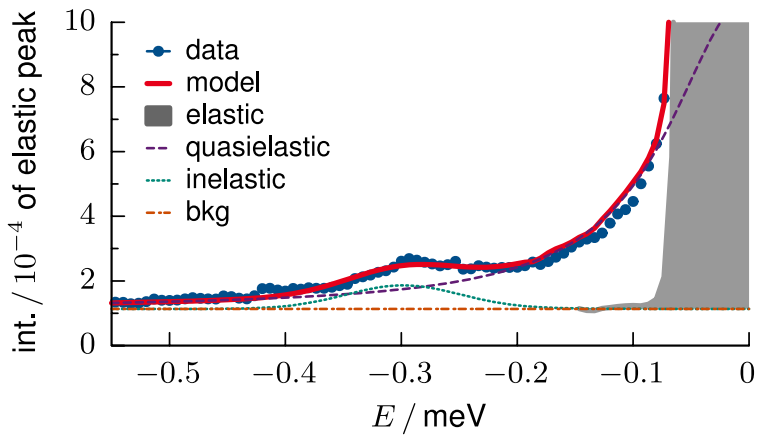

(b)

Figure 1. a) Inelastic neutron time of flight (IN5) scattering intensity on $\mathrm{PVFcI}_{x}$ at $T=80 \mathrm{~K}$ under external magnetic fields $B$. The inelastic contribution is attributed to electron Zeeman splitting of the oxidized ferrocene complex. Curves are vertically shifted for clarity. b) Model fit to data at $B=2.5 \mathrm{~T}$ with a quasielastic and an inelastic contribution.

further, we refer to the oxidized compound as $\mathrm{PVFcI}_{x}$. Powder samples were filled in hollow cylinder aluminum sample holders of $14 \mathrm{~mm}$ diameter and ca. $0.5 \mathrm{~mm}$ wall thickness.

Neutron spectroscopy was carried out on two different spectrometers at the Institut Laue-Langevin (ILL) in Grenoble, France. With the same incident wavelength of $6.3 \AA$ the energy resolution at the elastic line was $40 \mu \mathrm{eV}$ FWHM for the time of flight spectrometer IN5 and $0.8 \mu \mathrm{eV}$ FWHM for the backscattering spectrometer IN16. The sample was placed in an ILL standard superconducting cryomagnet on both instruments, allowing for vertical magnetic fields of up to $2.5 \mathrm{~T}$ to be applied at the sample position at temperatures from $2 \mathrm{~K}$ to $300 \mathrm{~K}$. The available vertical opening of $+-5^{\circ}$ restricted the scattering plane of observed scattered neutrons to be approximately perpendicular to the magnetic field. On IN16 therefore only about a quarter of the analyzer height was usable. In addition the large diameter of the sample environment made the use of the standard IN16 multidetector impossible. Instead, three single detectors were positioned to measure the scattered and energy analyzed neutron intensity for magnitudes of the scattering vector $Q$ of $0.85 \AA^{-1}, 1.26 \AA^{-1}$ and $1.6 \AA^{-1}$. Our experiment was the first and only cryomagnet experiment on IN16 ever.

The recorded data was treated by standard methods, subtracting empty cell scattering and correcting for angular dependent sample self absorption.

\section{Results and discussion}

\subsection{Zeeman splitting of electronic ground state}

The neutron time of flight spectra on $\mathrm{PVFcI}_{x}$ under magnetic fields revealed a very weak inelastic peak, four orders of magnitude less intense than the elastic peak. A shift of its position with changing field strength was observed as shown in Fig. 1a for $T=80 \mathrm{~K}$. The peak position appears to be independent of temperature between $2 \mathrm{~K}$ to $140 \mathrm{~K}$ and even up to $300 \mathrm{~K}$, but increasing quasielastic scattering from the ferrocene ring rotation is superimposed at $300 \mathrm{~K}$ complicating a reliable interpretation.
The neutron energy gain side of the spectra summed over all detectors was analyzed with a simple model function accounting for a delta-shaped elastic, Lorentzian quasielastic and Gaussian inelastic contribution. The sum is convoluted with the experimentally determined resolution function and constant background is added:

$$
\begin{aligned}
S(\omega)= & s_{0}\left[A_{0} \delta(\omega)+A_{1} \mathrm{e}^{\frac{\hbar \omega}{2 \mathrm{kB} T}} \mathcal{L}(\gamma, \omega)\right. \\
& \left.+C \mathcal{G}\left(\sigma, \omega+\omega_{0}\right)\right] \otimes \mathcal{R}(\omega)+\mathrm{bkg}
\end{aligned}
$$

where $A_{0}, A_{1}, C$ are the intensities of each contribution, $s_{0}$ an overall scaling factor, the exponential factor is the detailed balance, $\mathcal{L}$ a normalized Lorentzian of HWHM $\gamma$, $\mathcal{G}$ a normalized Gaussian of standard deviation $\sigma$, and $\mathcal{R}$ the experimentally determined instrumental resolution function. An exemplary fit is shown in Fig. $1 \mathrm{~b}$ at $T=80 \mathrm{~K}$ and $B=2.5 \mathrm{~T}$.

In contrast to the inelastic scattering, the quasielastic contribution was, within its uncertainty, independent of the applied field. Thus the Lorentzian width was fixed to the value obtained without external field in order to extract parameters of the inelastic contribution more reliably. The field dependency of the inelastic peak position $E_{0}=\hbar \omega_{0}$ is found to be linear as shown in top of Fig. 2. We assume that this peak arises from transitions between Zeeman split electronic ground state levels in the external field $B$ caused by an unpaired electron in the oxidized ferrocene complex. The Zeeman splitting is known to depend on $B$ according to

$$
E_{0}=\hbar \omega_{0}=g \mu_{B} B
$$

with the Bohr magneton $\mu_{B}$ and the Landé factor $g$. The slope of the linear fit in top Fig. 2 is $0.1185(8) \mathrm{meV} \mathrm{T}^{-} 1$, corresponding to $g=2.05(1)$. This value is only slightly larger than the spin-only value. Moreover, it differs significantly from the anisotropic $g$-factors of $g_{\|}=$ 4.35(5) and $g_{\perp}=1.26(6)$ obtained from electron spin resonance for ferrocenium ions in solution $[12,16]$, but these experiments also found decrease of the $g$-factor anisotropy for substituted ferrocenes. In fact, the excitation observed here is broader than the instrumental resolution, and the deconvoluted width $\sigma$ shows the tendency to increase with increasing field as shown in Fig. 2. This could mean that the obtained value of $g=2.05(1)$ is the 


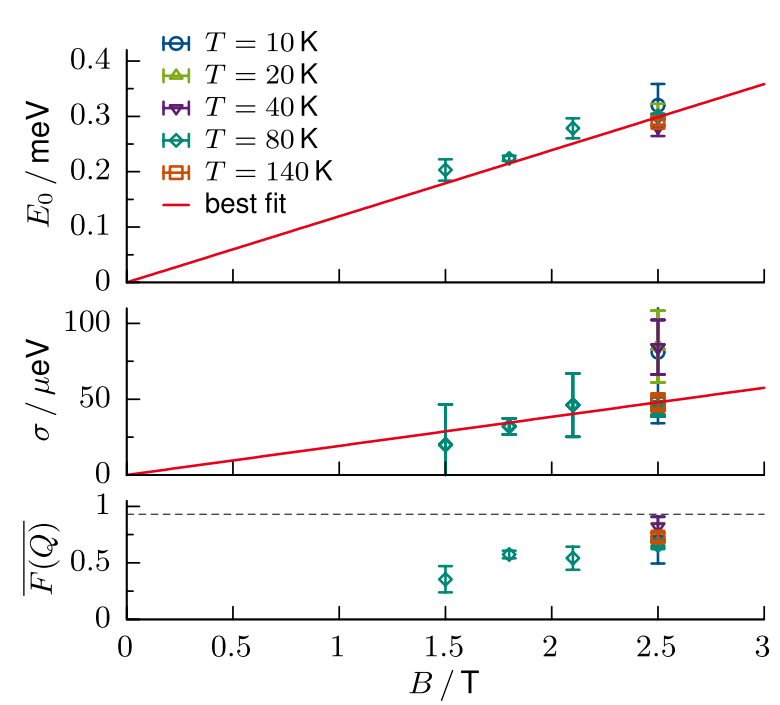

Figure 2. Obtained parameter values for energy $E_{0}$, unconvoluted width $\sigma$ and averaged magnetic form factor $\overline{F(Q)}$ of the inelastic excitation in $\mathrm{PVFcI}_{x}$ as function of applied magnetic field $B$.

mean value of a remaining, unresolved $g$-factor anisotropy. This anisotropy would be, however, less pronounced than in the ferrocenium ion, which could be explained by further delocalization of the electron through the attached polymer chain.

In order to estimate the intensity of the magnetic scattering, we calculated the double differential cross section for an uncorrelated spin- $\frac{1}{2}$ system in an external magnetic field from ref. [17, Eq. (7.27)]. Adding coherent and incoherent nuclear scattering the result helps to identify the contributions in Eq. (1) to

$$
\begin{aligned}
A_{0} & =\frac{\sigma_{\mathrm{coh}}}{4 \pi} \overline{S(Q)}+\frac{\sigma_{\mathrm{inc}}}{4 \pi} \overline{A_{0}^{\mathrm{inc}}(Q)}+\frac{\sigma_{\mathrm{mag}}}{4 \pi} \\
A_{1} & =\frac{\sigma_{\mathrm{inc}}}{4 \pi}\left(1-\overline{A_{0}^{\text {inc }}(Q)}\right) \\
C & =\frac{\sigma_{\mathrm{mag}}}{4 \pi}\left[1+\exp \left(\frac{\hbar \omega_{0}}{k_{\mathrm{B}} T}\right)\right]^{-1}
\end{aligned}
$$

with

$$
\frac{\sigma_{\mathrm{mag}}}{4 \pi}=\frac{1}{4} r_{0}^{2}\left(\frac{g}{2} \overline{F(Q)}\right)^{2}
$$

where $r_{0}^{2}=\left(\gamma e^{2} / m_{e} c^{2}\right)^{2}=0.29$ barn, $\overline{S(Q)}$ and $\overline{A_{0}^{\text {inc }}(Q)}$ are the static and elastic incoherent structure factor averaged over the probed Q-range respectively, and $\sigma_{\text {inc }}$ and $\sigma_{\text {coh }}$ are the nuclear incoherent and coherent scattering cross sections per formula unit. The intensity of the inelastic excitation originating from scattering of neutrons on magnetic moments is described by the magnetic scattering cross section in Eq. (6) and the thermal population factor in Eq. (5).

By using $\overline{S(Q)}=1.45$ obtained from a separate polarized neutron diffraction experiment [18], we calculated the magnetic form factor $\overline{F(Q)}$ of the oxidized ferrocene units averaged over the probed $Q$-range from $0.6 \AA^{-1}$ to $1.7 \AA^{-1}$. The resulting values are shown in bottom Fig. 2 . Unfortunately they are plagued by large uncertainties, but

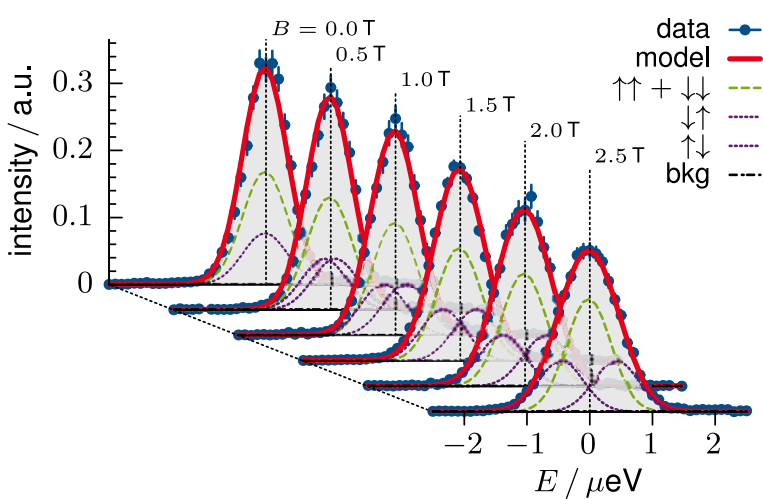

Figure 3. Broadening of the elastic peak in neutron backscattering spectroscopy (IN16) observed on $\mathrm{PVFcI}_{x}$ at $T=2 \mathrm{~K}$ under external magnetic fields $B$. The data was analyzed with a model accounting for hydrogen nuclear Zeeman splitting of non spinflip $(\uparrow \uparrow+\downarrow \downarrow)$ and spinflip scattering $(\downarrow \uparrow$ and $\uparrow \downarrow)$.

the values between ca. 0.6 to 0.8 could indicate that the electron is more delocalized than expected for a Fe $3 \mathrm{~d}$ shell (circa 0.92 [19], dashed line in Fig. 2). This is in qualitative agreement with studies of the electronic structure of ferrocene concluding that the highest occupied $e_{2}^{\prime}$ orbital consists of the $\mathrm{Fe} \mathrm{d}_{x^{2}-y^{2}}$ or $\mathrm{d}_{x y}$ shell mixed with a small contribution of the $\pi$ orbitals of the cyclopentadienyl ligands [20]. Moreover, it would agree with the possible explanation of further electron delocalization caused by the polymer chain leading to the reduced $g$-factor anisotropy. However, these results should be interpreted with great care as our evaluation of the magnetic scattering intensity is sensitive to the fraction of oxidized ferrocene units. They are expected to lie close to unity, but remain effectively unknown for our sample.

\subsection{Nuclear hyperfine splitting in the external field}

In high resolution neutron backscattering spectroscopy, a significant broadening of the elastic line was observed under external magnetic fields. Figure 3 shows data obtained for $\mathrm{PVFcI}_{x}$ at $T=2 \mathrm{~K}$ at different external field strengths. We believe that the observed broadening of the elastic line is caused by an unresolved splitting due to hyperfine splitting of the nuclear ground state of hydrogen in the external magnetic field. An incoherent scattering process with spin flip of the neutron changes the orientation of the hydrogen spin relative to the quantization axis by $\Delta m_{I}= \pm 1$, thus changing its energy by a magnitude of

$$
E_{0}=\hbar \omega_{0}=g_{I} \mu_{N} B
$$

The double differential scattering cross section can be written in the form as derived by Heidemann [13]

$$
\begin{aligned}
\frac{\mathrm{d}^{2} \sigma}{\mathrm{d} \Omega \mathrm{d} \omega}=s_{0} \cdot[ & \left(\frac{\sigma_{\mathrm{coh}}}{\sigma_{\text {inc }}} \overline{S(Q)}+\frac{1}{3}\right) \delta(\omega)+\frac{1}{3} \delta\left(\omega-\omega_{0}\right) \\
& \left.+\frac{1}{3} \delta\left(\omega+\omega_{0}\right)\right] \otimes \mathcal{R}(\omega)+\text { bkg }
\end{aligned}
$$


Table 1. Resulting Landé factors of nuclear magnetic moment $g_{\mathrm{I}}$ and values used as average structure factor $\overline{S(Q)}$.

\begin{tabular}{|c|c|c|c|}
\hline sample & $T / K$ & $\overline{S(Q)}$ & $g_{\mathrm{I}}$ \\
\hline $\operatorname{PVFcI}_{x}$ & 2 & 1.52 & $5.81(4)$ \\
\hline $\operatorname{PVFcI}_{x}$ & 20 & 1.52 & $5.74(5)$ \\
\hline $\operatorname{PVFcI}_{x}$ & 40 & 1.52 & $5.60(5)$ \\
\hline $\mathrm{FcI}_{3}$ & 10 & 1.6 & $5.05(8)$ \\
\hline $\mathrm{Fc}$ & 2 & 1.81 & $5.96(8)$ \\
\hline Reference value for ${ }^{1} \mathrm{H}$ : & & & $5.58^{\mathrm{a}}$ \\
\hline Vanadium & 2 & 0 & $1.1(5)$ \\
\hline Reference value for ${ }^{51} \mathrm{~V}$ : & & & $1.47^{\mathrm{a}}$ \\
\hline
\end{tabular}

to which we have added an elastic coherent contribution using the Q-averaged static structure factor $\overline{S(Q)}$. $\mathcal{R}(\omega)$ is a Gaussian with the fixed width of the experimental resolution function obtained without magnetic field. The incoherent part of the scattering splits up in 3 peaks of equal intensity located at $E=0$ and $\pm E_{0}$. With values of $\overline{S(Q)}$ obtained from a polarized neutron diffraction experiment [18], Eq. (8) was fitted to the data as shown in Fig. 3. The refinement was performed simultaneously for spectra obtained at different fields using $g_{\text {I }}$ as free parameter. The values used for $\overline{S(Q)}$ and the resulting values of $g_{\mathrm{I}}$ are given in Table 1. For the samples $\mathrm{PVFcI}_{x}, \mathrm{FcI}_{3}$ and $\mathrm{Fc}$ virtually all incoherent scattering originates from hydrogen. The proximity of the results to the reference value of $g_{I}=5.58$ [21] confirms that the observed line broadening is caused by the interaction of hydrogen nuclear spins with the external magnetic field. However, the agreement within the given uncertainties is moderate indicating a larger uncertainty of systematic origin, for example field inhomogeneities. But given that the splitting lies within the resolution function, the result is satisfactory and demonstrates the sensitivity of the experimental high resolution technique to external magnetic fields.

Table 1 also shows the result obtained for a vanadium sheet. Due to the small Lande factor of the vanadium nuclear spin the broadening is barely distinguishable in the present setup, but the result corresponds within its large uncertainty to the reference value of $g_{I}=1.47$ [21].

\section{Conclusion}

We presented neutron spectroscopy experiments observing the ground state splitting in external magnetic fields on electronic and nuclear magnetic moments. The spectrum of the oxidized redox-responsive polymer poly(vinylferrocene) showed an inelastic excitation which we assume to be caused by splitting of the paramagnetic electronic ground state of the oxidized ferrocene units, with a Landé factor of $g=2.05(1)$. The results indicate that this could be the averaged value of an unresolved $g$-factor anisotropy, which would however be less pronounced than in the ferrocenium ion. In a second experiment, the nuclear hyperfine splitting under external magnetic fields caused significant broadening of the elastic line in incoherent neutron backscattering spectroscopy, where the analysis showed consistency with the nuclear $g_{\mathrm{I}}$-factors for hydrogen and vanadium.

The Institut Laue-Langevin (Grenoble, France) is acknowledged for the allocation of beamtime on IN5 and IN16. The authors would like to thank Jacques Ollivier for his help and support during the experiment on IN5, and are grateful for valuable comments on the manuscript from Gøran Nilsen and an unknown referee. J. E., M. G. and B. S. thank the Landes-Offensive zur Entwicklung Wissenschaftlichökonomischer Exzellenz (LOEWE Soft Control) for ongoing financial support of this work.

\section{References}

[1] M.A.C. Stuart, W.T.S. Huck, J. Genzer, M. Müller, C. Ober, M. Stamm, G.B. Sukhorukov, I. Szleifer, V.V. Tsukruk, M. Urban et al., Nat. Mater. 9, 101 (2010)

[2] M. Ramanathan, Y.C. Tseng, K. Ariga, S.B. Darling, J. Mater. Chem. C 1, 2080 (2013)

[3] E. Lallana, N. Tirelli, Macromol. Chem. Phys. 214, 143 (2013)

[4] G.R. Whittell, M.D. Hager, U.S. Schubert, I. Manners, Nat. Mater. 10, 176 (2011)

[5] M. Nakahata, Y. Takashima, H. Yamaguchi, A. Harada, Nat. Commun. 2, 511 (2011)

[6] J. Elbert, M. Gallei, C. Rüttiger, A. Brunsen, H. Didzoleit, B. Stühn, M. Rehahn, Organometallics 32, 5873 (2013)

[7] J. Elbert, F. Krohm, C. Rüttiger, S. Kienle, H. Didzoleit, B.N. Balzer, T. Hugel, B. Stühn, M. Gallei, A. Brunsen, Adv. Funct. Mater. 24, 1591 (2013)

[8] A. Gardner, J. Howard, T. Waddington, R. Richardson, J. Tomkinson, Chem. Phys. 57, 453 (1981)

[9] E. Kemner, M. de Schepper, A. Schmets, H. Grimm, A. Overweg, R. van Santen, J. Phys. Chem. B 104, 1560 (2000)

[10] M. Hmyene, A. Yassar, M. Escorne, A. PercheronGuegan, F. Garnier, Adv. Mater. 6, 564 (1994)

[11] Y. Ando, T. Hiroike, T. Miyashita, T. Miyazaki, Thin Solid Films 350, 232 (1999)

[12] S.E. Anderson, R. Rai, Chem. Phys. 2, 216 (1973)

[13] A. Heidemann, Z. Phys. 238, 208 (1970)

[14] T. Chatterji, B. Frick, H.S. Nair, J. Phys.: Condens. Matter 24, 266005 (2012)

[15] M. Gallei, R. R Klein, M. Rehahn, Macromolecules 43, 1844 (2010)

[16] R. Prins, Mol. Phys. 19, 603 (1970)

[17] S. Lovesey, Theory of neutron scattering from condensed matter, Vol. 2 (Clarendon Press, 1984)

[18] M. Appel, B. Frick, J. Elbert, M. Gallei, B. Stühn, to be published

[19] P.J. Brown, Magnetic form factors (John Wiley \& Sons, Ltd, 2006), Vol. C of International Tables for Crystallography, chap. 4.4.5, pp. 454-461

[20] Z.F. Xu, Y. Xie, W.L. Feng, H.F. Schaefer, J. Phys. Chem. A 107, 2716 (2003)

[21] R.B. Firestone, Table of Isotopes, 8th edn. (WileyVCH, Berlin, 1999) 